\title{
Avaliação do potencial quimioprotetor do fruto de Psidium guajava contra os efeitos genotóxicos da doxorrubicina
}

\section{Evaluation of Psidium guajava fruit chemoprotective against the genotoxic effects of doxorubicin}

Silvio de Almeida Junior ${ }^{1}$ (D), Gabriel Nalini Branquinho², Lidiane Andrade Silva Santos ${ }^{2}$, Lucas Henrique Domingos da Silva², Roberta Cristina Ribeiro Cruz², Fernanda Gosuen Gonçalves Dias'1, Ricardo Andrade Furtado'

'Programa de Pós-graduação em Ciência Animal, Universidade de Franca - Franca (SP), Brasil.

2Universidade de Franca - Franca (SP), Brasil.

Como citar: Almeida Junior S, Branquinho GN, Santos LAS, Silva LHD, Cruz RCR, Dias FGG, et al. Avaliação do potencial quimioprotetor do fruto de Psidium guajava contra os efeitos genotóxicos da doxorrubicina. Cad Saúde Colet, 2020;28(3):422-425. https://doi.org/10.1590/1414-462X202028030501

\section{Resumo}

Introdução: A goiaba é um fruto amplamente utilizado como alimento e é considerada planta medicinal em países tropicais e subtropicais. Pesquisas têm mostrado que o fruto contém constituintes químicos com abrangente uso clínico. Além disso, a maior parte das substâncias utilizadas no tratamento contra câncer foi isolada a partir de produtos naturais. Objetivo: Avaliar o potencial citotóxico, mutagênico, antimutagênico e quimioprotetor da fruta liofilizada de Psidium guajava, a goiaba, in vivo. Método: A citotoxicidade, a mutagenicidade e a antimutagenicidade foram avaliadas em três diferentes dosagens $(0,625,1,25$ e 2,50 g/kg) de goiaba. Resultados: Os resultados mostraram que a goiaba não apresentou atividade citotóxica e mutagênica no ensaio de micronúcleo em sangue periférico e que não houve alterações nos valores de ALT e AST, indicando ausência de toxicidade hepática. Nos animais tratados com a goiaba, a dose de $0,625 \mathrm{mg} / \mathrm{kg}$ significativamente reduziu os danos induzidos pela doxorrubicina. Conclusão: Esses resultados mostraram que o consumo de goiaba é seguro e capaz de proteger o material genético de alterações genômicas.

Palavras-chave: goiaba; teste do micronúcleo; doxorrubicina.

\begin{abstract}
Background: Guava is a fruit widely used as food and is considered a medicinal plant in the tropical and subtropical countries. Scientific research has shown that the fruit contains chemical constituents with comprehensive clinical use. In addition, most of the substances used in cancer treatment have been isolated from natural products. Objective: To evaluate the cytotoxic, mutagenic, antimutagenic, and chemoprotective potential of the freeze-dried fruit of Psidium guajava, guava, in vivo. Method: Cytotoxicity, mutagenicity, and antimutagenicity were evaluated in three different dosages $(0.625,1.25,2.50 \mathrm{~g} / \mathrm{kg}) \mathrm{of}$ guava. Results: The results show that guava does not present cytotoxic 2 and mutagenic activity in the micronucleus assay in peripheral blood and there were no alterations in ALT and AST values showing the absence of hepatic toxicity. In animals treated with guava, the dose of $0.625 \mathrm{mg} / \mathrm{kg}$ significantly reduced the damage induced by doxorubicin. Conclusion: These results show that guava consumption is safe as it is also capable of protecting the genetic material from changes.
\end{abstract}

Keywords: guava; micronucleus test; doxorubicin.

Trabalho realizado na Universidade de Franca - Franca (SP), Brasil.

Correspondência: Ricardo Andrade Furtado. E-mail: ricardo.furtado@unifran.edu.br

Conflito de interesses: nada a declarar.

Recebido em: Nov. 01, 2018. Aprovado em: Out. 13, 2019

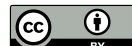

Este é um artigo publicado em acesso aberto (Open Access) sob a licença Creative Commons Attribution, que permite uso, distribuição e reprodução em qualquer meio, sem restrições desde que o trabalho original seja corretamente citado. 


\section{INTRODUÇÃO}

A fruta de Psidium guajava (goiaba) se desenvolve em regiões tropicais e subtropicais, preferencialmente em clima seco, possuindo polpa suculenta, doce-acidulada e aromática, com numerosas sementes pequenas e duras, contendo na composição $13,2 \%$ de carboidratos, 0,88\% de proteínas, 0,53\% de lipídeos e $84,9 \%$ de água'.

A fruta de $P$. guajava foi selecionada por ser amplamente consumida, comum em países tropicais e subtropicais e utilizada na medicina popular². Entre suas atividades biológicas pode-se citar os efeitos antinociceptivo, analgésico ${ }^{3}$ e hepatoprotetor ${ }^{4}$. Essas atividades foram atribuídas pela presença, principalmente, de compostos fenólicos, flavonoides, carotenoides, terpenoides e triterpenos ${ }^{2}$.

Diante das propriedades medicinais descritas ${ }^{2-4}$, o objetivo do trabalho foi avaliar o potencial citotóxico e mutagênico da goiaba em células de camundongos, bem como avaliar o efeito quimioprotetor da goiaba contra dados causados pela doxorrubicina.

\section{MÉTODO}

\section{Obtenção e seleção da fruta de Psidium guajava}

As frutas de Psidium guajava (variedade Pedro Sato lisa) foram adquiridas de forma comercial na cidade de Franca, São Paulo, Brasil, em ponto de amadurecimento médio e com média de peso médio de $157 \mathrm{~g}$. Para experimentação, elas foram submetidas ao processo de liofilização no Laboratório de Ciências da Universidade de Franca e, posteriormente, mantidas em freezer $\left(-20^{\circ} \mathrm{C}\right)$ até seu uso.

\section{Delineamento experimental}

Foram utilizados 30 camundongos Swiss machos (Mus musculus), com idade de 6 a 8 semanas e peso médio de $30 \mathrm{~g}$, adquiridos por meio do Biotério Central da Universidade de São Paulo, Ribeirão Preto (São Paulo, Brasil). O protocolo de trabalho foi aprovado pela Comissão de Ética no Uso de Animais da Universidade de Franca (CEUA - protocolo 458301116). Os roedores foram aleatoriamente separados nos grupos experimentais $(n=5)$ : controle negativo (água filtrada), controle positivo (DXR $10 \mathrm{mg} / \mathrm{kg}$ intraperitoneal), goiaba $(2,5 \mathrm{mg} / \mathrm{kg}$ ) e três diferentes doses da fruta $(0,625,1,25$ e $2,5 \mathrm{~g} / \mathrm{kg})$ associadas à doxorrubicina ${ }^{\circledR}$ (DXR). O estudo foi realizado nos anos de 2017-2018.

Os diferentes tratamentos foram administrados por dois dias consecutivos. Após o período de $24 \mathrm{~h}$ após o último tratamento, os animais foram eutanasiados com tiopental sódico $(0,83 \mathrm{~g} / \mathrm{kg}$, por via intraperitoneal), e imediatamente foi realizada punção cardíaca para coleta de sangue.

\section{Ensaios}

O micronúcleo de sangue periférico foi realizado de acordo com o protocolo descrito na OECD 4745. A razão de eritrócitos imaturos (EIM) pelo total de eritrócitos foi calculada pela análise de 2.000 eritrócitos para determinar a citotoxicidade da fruta. Foi analisado um total de 5.000 eritrócitos imaturos (EIM) por animal para a determinação da frequência de eritrócitos imaturos micronucleados (EIM-MN). As lâminas foram analisadas em estudo cego em microscopia de luz com 1.000x de aumento.

Com o plasma, foram realizadas dosagens séricas de alanina aminotransferase (ALT) e aspartato aminotransferase (AST) para avaliação de dano hepático.

Os resultados foram analisados estatisticamente por análise de variância (ANOVA) e comparados pelo método de Tukey, e a diferença mínima significante foi calculada para $a=0,05$.

\section{RESULTADOSE DISCUSSÃO}

Na Tabela 1 estão expressas as médias das frequências de EIM-MN e a razão de EIM pelo total de eritrócitos. Os resultados demonstraram que não houve diferença estatisticamente na frequência de micronúcleos em células de sangue periférico entre o grupo tratado com 
goiaba na dose de $2,5 \mathrm{~g} / \mathrm{kg}$ isolada com o grupo controle negativo. Esses achados são suficientes para indicar a ausência de efeito mutagênico da dose testada de goiaba no presente trabalho ${ }^{5}$. O grupo controle positivo apresentou diferença estatística quando comparado ao controle negativo, conforme esperado. A administração das doses de goiaba $(0,625 \mathrm{~g} / \mathrm{kg})$ reduziu em $30,7 \%$ os danos induzidos por DXR de maneira estatística, revelando atividade antigenotóxica da fruta da goiabeira.

Tabela 1. Frequências de eritrócitos imaturos micronucleados (EIM-MN) e razão EIM/total de eritrócitos obtidos em sangue periférico de camundongos tratados com diferentes doses de goiaba associadas ou não à DXR. Franca, São Paulo, Brasil, 2017/2018

\begin{tabular}{lcc} 
Tratamentos (g/kg) & $\begin{array}{c}\text { EIM-MN } \\
\text { (Média } \pm \text { desvio-padrão) }\end{array}$ & $\begin{array}{c}\text { EIM/ total de eritrócitos } \\
\text { (Média } \pm \text { desvio-padrão) }\end{array}$ \\
\hline Controle negativo & $31,4 \pm 3,9$ & $0,051 \pm 0,011$ \\
\hline Goiaba 2,5 & $34,0 \pm 2,9$ & $0,057 \pm 0,006$ \\
\hline Controle positivo & $88,6 \pm 15,0^{\mathrm{b}}$ & $0,073 \pm 0,014$ \\
\hline Goiaba 0,625 + DXR & $61,4 \pm 4,7^{\mathrm{a}, \mathrm{b}}$ & $0,075 \pm 0,032$ \\
\hline Goiaba 1,25 + DXR & $89,0 \pm 15,4^{\mathrm{b}}$ & $0,063 \pm 0,015$ \\
\hline Goiaba 2,5 + DXR & $72,6 \pm 8,0^{\mathrm{b}}$ & $0,051 \pm 0,013$ \\
\hline
\end{tabular}

Controle negativo: água filtrada (veículo); Controle positivo: doxorrubicina (DXR, $10 \mathrm{mg} / \mathrm{kg}$ ). ${ }^{\mathrm{a}}$ Significantemente diferente

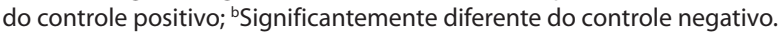

A Tabela 1 também mostra a razão de EIM pelo total de eritrócitos e seu respectivo desvio-padrão obtido para todos os grupos de tratamento. Nenhuma redução significante na porcentagem de EIM em relação ao total de eritrócitos foi observada para nenhum grupo comparado ao controle negativo, demonstrando a ausência de citotoxicidade dos diferentes tratamentos nas presentes condições experimentais. Quanto à toxicidade hepática, avaliada pelos níveis séricos de ALT e AST, no estudo foi comprovado que a dosagem administrada de $2,5 \mathrm{~g} / \mathrm{kg}$ de goiaba não apresentou diferenças significativas do controle negativo (Tabela 2), mostrando ausência de hepatotoxicidade.

Tabela 2. Avaliação de hepatotoxicidade por meio de análise sérica de alanina aminotransferase (ALT) e aspartato aminotransferase (AST) de camundongos Swiss tratados com goiaba. Franca, São Paulo, Brasil, 2017/2018

\begin{tabular}{lcc}
\multicolumn{1}{c}{ Tratamentos (g/kg) } & ALT (Média \pm desvio-padrão) & AST (Média \pm desvio-padrão) \\
\hline Controle negativo & $66,6 \pm 3,71$ & $141,0 \pm 14,27$ \\
\hline Goiaba 2,5 & $61,6 \pm 11,95$ & $80,5 \pm 11,68$ \\
\hline Controle positivo (DXR-10) & $64,8 \pm 3,90$ & $143,6 \pm 9,04$ \\
\hline
\end{tabular}

Controle negativo: água filtrada (veículo); Controle positivo: doxorrubicina (DXR, $10 \mathrm{mg} / \mathrm{kg}$ ).

Os dados encontrados no presente estudo são corroborados pelos achados por Teixeira et al. ${ }^{6}$, os quais mostraram ausência de efeito mutagênico, in vivo e in vitro da goiaba, pelos testes em micronúcleo em medula óssea de ratos e em linfócitos humanos.

Os resultados do presente trabalho podem ser atribuídos à atividade antioxidante de compostos sabidamente encontrados na fruta. A presença de flavonoides como ácido ascórbico e licopeno ${ }^{7}$ pode ser o responsável pela atividade observada no presente estudo, pois suas atividades biológicas têm sido bem correlacionadas com efeitos quimiopreventivos, como atividades antitumoral e anti-inflamatória e efeito citotóxico em relação a tumores em ensaios in vitro ${ }^{8-10}$.

Em conclusão, por se tratar de uma fruta altamente consumida pela população por via oral, com os dados encontrados é possível confirmar que a goiaba não apresenta toxicidade 
hepática e citotoxicidade, assim como apresenta atividade quimioprotetora contra os efeitos mutagênicos induzidos pela administração da doxorrubicina em camundongos, possivelmente atribuídos aos compostos presentes no fruto.

\section{REFERÊNCIAS}

1. Medina LM, Pagano FG. Caracterización de la pulpa de guayaba (Psidium guajava L.) tipo "Criolla Roja". Rev Fac Agron. 2003;20(1):72-86.

2. Gutiérrez RMP, Mitchell S, Solis RV. Psidium guajava: a review of its traditional uses, phytochemistry and pharmacology. J Ethnopharmacol. 2008;117(1):1-27. http://dx.doi.org/10.1016/j.jep.2008.01.025. PMid:18353572.

3. Kakuo S, Fushimi T, Kawasaki K, Nakamura J, Ota N. Effects of Psidium guajava Linn. leaf extract in Japanese subjects with knee pain: a randomized, double-blind, placebo-controlled, parallel pilot study. Aging Clin Exp Res. 2018;30(11):1391-8. http://dx.doi.org/10.1007/s40520-018-0953-6. PMid:29687303.

4. Saber FR, Abdelbary GA, Salama MM, Saleh DO, Fathy MM, Soliman FM. UPLC/QTOF/MS profiling of two Psidium species and the in-vivo hepatoprotective activity of their nano-formulated liposomes. Food Res Int. 2018;105:1029-38. http://dx.doi.org/10.1016/j.foodres.2017.12.042. PMid:29433194.

5. Organisation for Economic Cooperation and Development. Test No. 474: mammalian erythrocyte micronucleus test [Internet]. [citado em 2018 set 10]. Disponível em: https://www.oecd-ilibrary.org/ environment/test-no-474-mammalian-erythrocyte-micronucleus-test_9789264224292-en

6. Teixeira RO, Camparoto ML, Mantovani MS, Vicentini VEP. Assessment of two medicinal plants, Psidium guajava L. and Achillea millefolium L., in in vitro and in vivo assays. Genet Mol Biol. 2003;26(4):551-5. http:// dx.doi.org/10.1590/S1415-47572003000400021.

7. Zhao Y-J, Li J-K, Zhang X, Gao J-P. Study on antioxidant activity of flavonoids from leaves of Psidium guajava. Zhongguo Zhong Yao Za Zhi. 2018;43(4):760-5. PMid:29600652.

8. Santos RC, Ombredane AS, Souza JMT, Vasconcelos AG, Plácido A, Amorim ADGN, et al. Lycopene-rich extract from red guava (Psidium guajava L.) displays cytotoxic effect against human breast adenocarcinoma cell line MCF-7 via an apoptotic-like pathway. Food Res Int Ott Ont. 2018;105:184-96. http://dx.doi. org/10.1016/j.foodres.2017.10.045. PMid:29433206.

9. Qin X-J, Yu Q, Yan H, Khan A, Feng M-Y, Li P-P, et al. Meroterpenoids with antitumor activities from guava (Psidium guajava). J Agric Food Chem. 2017;65(24):4993-9. http://dx.doi.org/10.1021/acs.jafc.7b01762. PMid:28578580.

10. Vasconcelos AG, Amorim ADGN, Santos RC, Souza JMT, de Souza LKM, Araújo TSL, et al. Lycopene rich extract from red guava (Psidium guajava $\mathrm{L}$.) displays anti-inflammatory and antioxidant profile by reducing suggestive hallmarks of acute inflammatory response in mice. Food Res Int. 2017;99(Pt 2):959-68. http:// dx.doi.org/10.1016/j.foodres.2017.01.017. PMid:28847433. 